Kaur Alttoa

\title{
DIE KAPITELLE DER ORDENSBURG FELLIN (VILJANDI) - DINGE AUS ZWEITER HAND AUS ALT-PERNAU (VANA-PÄRNU)?
}

Die Wiederentdeckung der im Nordischen Krieg gründlich zerstörten Ordensburg Fellin fällt in die Jahre 1878/79, als hier umfangreiche Ausgrabungen erfolgten. ${ }^{1}$ Diese wurden vom Felliner Gymnasiallehrer Theodor Schiemann (1847-1921) geleitet, später Professor für Osteuropäische Geschichte an der Friedrich-Wilhelms-Universität zu Berlin und Mitbegründer der deutschen Osteuropaforschung. Leider erfolgten nach den Ausgrabungen keinerlei Konservierungsarbeiten, weswegen ein großer Teil der damals gesäuberten, interessanten baulichen Details im Laufe der Zeit vernichtet wurden und erhalten blieben hauptsächlich Stümpfe. Bedauerlicherweise sind auch die die Ausgrabungsarbeiten dokumentierenden Materialien überaus knapp. Regulär berichtete die örtliche Zeitung, der „Felliner Anzeiger ${ }^{\prime 2}$, über die Arbeiten. Im Museum Viljandi wird auch das

DOI: https://doi.org/10.12697/BJAH.2017.13.02

Übersetzung aus dem Estnischen von Marju und Olaf Mertelsmann.

Der größte Teil der in diesem Beitrag vorgestellten Illustrationen (darunter die meisten Fotos Kaur Alttoas) stammen aus dem Viljandi Museum. Der Autor dankt Jaak Pihlak, Ain Vislapuu und Herki Helves für die Möglichkeit, diese Materialien hier zu nutzen.

1 Aare Kodar, „Esimesed arheoloogilised väljakaevamised Viljandi linnusevaremetes 1878.1879. a.“, Viljandi Muuseumi aastaraamat 1997 (Viljandi: Viljandi Muuseum, 1998), 20-33.

2 Weiterhin: FA. 


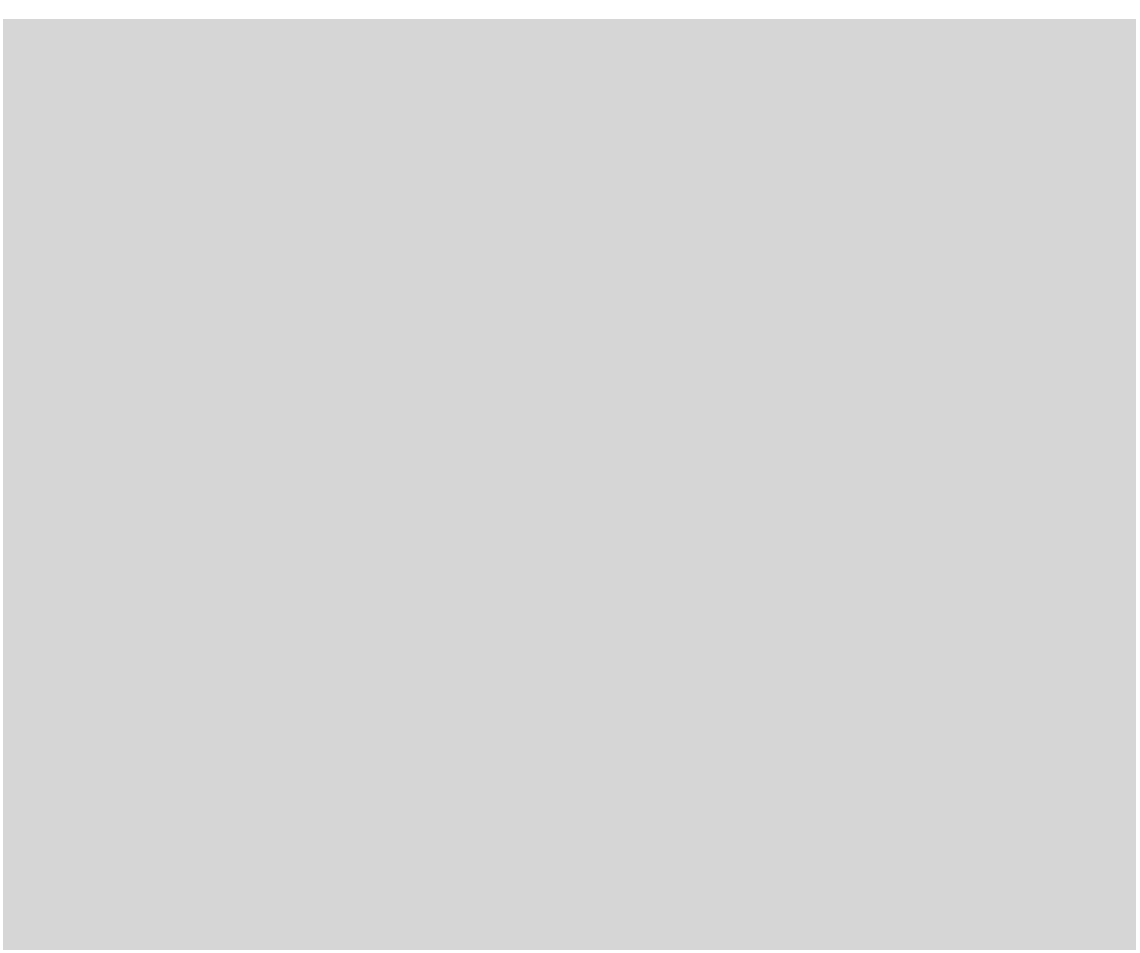

Abb. 1. Ausgrabungen 1878 in der Ordensburg Fellin. An zwei Seiten des Hofes sind Reste der Pfeiler des Kreuzgangs zu sehen. Foto: Theodor John, Viljandi Museum.

Tagebuch der Ausgrabungen aufbewahrt. ${ }^{3}$ Neben der Buchhaltung und den Arbeitsanweisungen finden sich dort knappe Angaben über die Ergebnisse der Grabungen, doch größtenteils sind diese Informationen schwer interpretierbar. Theodor Schiemann plante wohl, eine umfassendere Zusammenfassung der Ergebnisse vorzulegen. Tatsächlich ist von ihm jedoch nur ein einziger Zeitungsartikel über die Ausgrabungen erschienen, der hauptsächlich über das gefundene Material berichtet. ${ }^{4}$ Einiges mehr an Informationen bietet der vom Ausgrabungscomité vorgestellte zusammenfassende Überblick in der Zeitung, in dem die ausgegrabenen Bauteile beschrieben werden. ${ }^{5}$ Irgendwelche Pläne oder Zeichnungen, welche die Ausgrabungsergebnisse widerspiegeln, sind uns nicht überliefert

3 Viljandi Museum [Viljandi Muuseum, VM], 3413.

4 Theodor Schiemann, „Bericht über die Ausgrabungen bei der Schloßruine“, FA, Nr. 44 (1878)

5 FA, Nr. 42 (1878).

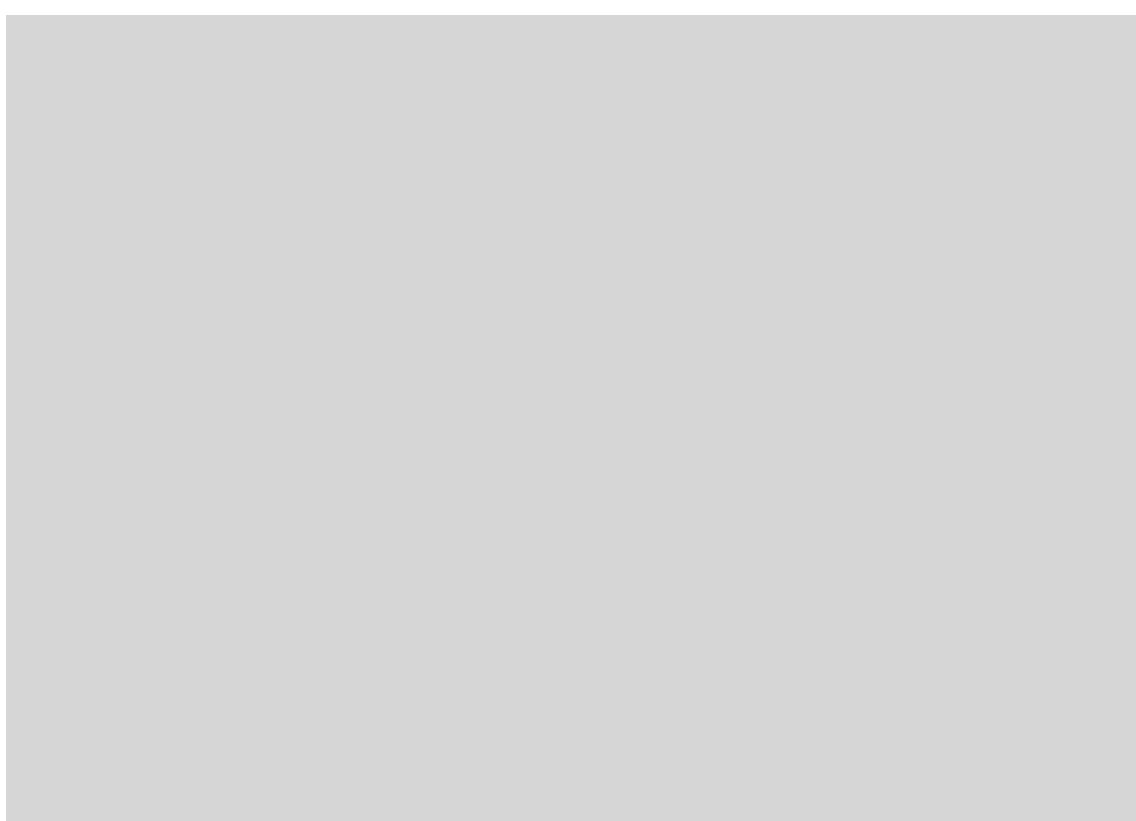

Abb. 2. Die bei der Ausgrabung 1878 entdeckten Funde. Foto: Theodor John, Viljand Museum.

worden, bekannt ist nur dies, dass der Landvermesser Ernst Bloßfeld für einen von den Ausgrabungen angefertigten Plan 50 Rubel erhielt. ${ }^{6}$ Einen ersten Rekonstruktionsplan veröffentlichte Karl von Löwis of Menar erst im Jahr 1922. ${ }^{7}$ Wohl sind mehrere Details auf der Zeichnung nicht in Übereinstimmung mit den natürlichen Angaben - so erscheint es als offensichtlich, dass in diesem Fall keine Vermessungszeichnung aus der Zeit der Ausgrabung als Grundlage diente, sondern eine später angefertigte Skizze.

\section{DAS KONVENTHAUS IN FELLIN UND DIE PROBLEME MIT SEINER DATIERUNG}

Der Kern der Burg Fellin war ein Konventhaus, bei dem sich die vier Gebäudeflügel rund um einen Innenhof mit einem quadratförmigen Grundriss befanden. Von den Ausmaßen her blieb dieses Haus ein

\footnotetext{
6 FA, Nr. 28 und 43 (1878).

7 Karl von Löwis of Menar, Burgenlexikon für Alt-Livland (Riga: Walters und Rapa, 1922).
} 
wenig kleiner als das in der Burg Riga (Länge der Seite entsprechend etwa 54,5 und ca. 57 Meter). Die einzelnen Flügel des Gebäudes verband auf der Hofseite ein Kreuzgang. In der nordwestlichen Ecke befand sich ein rechteckige Turm, der Lange Hermann. Vom Konventhaus ist einzig die westliche Wand und teilweise Mauerwerk des Untergeschosses erhalten geblieben. Wichtige Informationen über die seinerzeitige Gestalt der Burg bietet die von den Polen erstellte, detaillierte Revision der Anlage. ${ }^{8}$

Zeitgenössisches, schriftliches Material über die mittelalterliche Bautätigkeit in Estland ist überaus knapp. Dies gilt auch für die Ordensburg Fellin. Dank einer seltenen Information wissen wir über den Baubeginn der steinernen Gebäude in der Burg Fellin so gibt nämlich die Chronik Hermann Wartberges bekannt, dass Ordensmeister Volquin hier im Jahr 1224 anfing, eine Steinburg zu errichten. ${ }^{9}$ Leider ist dies auch die einzige Angabe über die Baugeschichte der Burg in der Ordenszeit. Über den Bau des Konventhauses fehlen jegliche historische Informationen. In einer solchen Situation haben Architekturhistoriker so manches Mal die Typologie zu Hilfe genommen. Derartig ist auch Armin Tuulse verfahren. Damals war das letzte Wort zur Untersuchung des Konventhauses die 1927 erschienene Monografie von Karl Heinz Clasen über preußische Burgen..$^{10}$ Beim Versuch, das dort vorgestellte Entwicklungsschema eines Konventhauses mit den in Fellin aufgefunden Bauskulpturen zu vereinen, kam Tuulse zu der Schlussfolgerung, dass mit dem Bau des Konventhauses in Fellin um das Jahr 1300 herum begonnen wurde. ${ }^{11}$ Dieser Standpunkt blieb mit kleinen Variationen für eine lange Zeit in der estnischen architektonischen Literatur dominierend: beispielsweise hat Villem Raam die Errichtung des Konventhauses auf das Ende des 13. und den

8 Polska XVI wieku pod wzgledem geograficzno-statystycznym. T. XIII, Inflanty, Cz. 1, hrsg. von Jan Jakubowski, Joźef Kordzikowski (Warszawa: Gbethner i Wolff, 1915), 162-179. Estnische Katrin Vabamäe, kommentiert von Kaur Alttoa (Viljandi: Viljandi Muuseum, 1998), 146-171. 9 „Hermanni de Wartberge Chronicon Livoniae“, Scriptores rerum Prussicarum II, hrsg. von Ernst Strehlke (Leipzig: Hirzel, 1865), 4

10 Karl Heinz Clasen, Die mittelalterliche Kunst im Gebiete des Deutschordensstaates Preußen. Die Burgbauten (Königsberg in Pr.: Gräfe und Unzer, 1927).

11 Armin Tuulse, Die Burgen in Estland und Lettland. Verhandlungen der Gelehrten Estnischen Gesellschaft XXXIII (Dorpat: Dorpater Estnischer Verlag, 1942), 143.
Beginn des 14. Jahrhunderts verortet. ${ }^{12}$ Der heutige Forschungsstand hat das Bild eher undeutlicher gemacht. Die fortgesetzte Untersuchung des Konventhauses als eines Burgentyps hat zu der Folgerung geführt, dass hier sichere typologische Erkennungszeichen fehlen, auf deren Grundlage sich eine Burg einzig auf Grundlage des Planungsschemas mit einem Vierteljahrhundert Genauigkeit datieren ließe. Im Moment kann nur zusammengefasst werden, dass die Herausbildung eines Konventhauses - hier ist von einer regulären Anlage mit vier ausgebauten Flügeln die Rede - in Preußen in den Zeitraum von etwa 1280-1300 fiel und dass dieser Burgentyp in der "klassischen“ Form sich in den Jahren 1300-1330 verbreitete. ${ }^{13}$ Ungefähr in dieses halbe Jahrhundert sollte auch der Bau des Konventhauses in Fellin gefallen sein. Etwas Genaueres lässt sich leider nicht sagen. Auch die Angaben der archäologischen Untersuchungen der letzten zwei Jahrzehnte bieten keine Hilfe: auf Basis des Fundmaterials lässt sich das Konventhaus auf das letzte Drittel des 13. Jahrhunderts oder den Beginn des 14. Jahrhunderts datieren. ${ }^{14}$

Im Falle des Konventhauses Fellin muss als Exkurs auf den in der nordwestlichen Ecke der Burg befindlichen Turm Langer Hermann eingegangen werden. Dieser verfügte über einen tiefen Kellerraum und sieben Stockwerke. Der Turm liegt vor den äußeren Konturen des Konventhauses. Unter Berücksichtigung dieser Flankierung interpretierte Armin Tuulse ihn als sekundäre Ergänzung des Konventhauses und behauptete, dass der Turm nicht vor der Mitte des 14. Jahrhunderts errichtet wurde. ${ }^{15}$ Im Verlauf der 1971 erfolgten Ausgrabungen war es möglich, den Verbindungsort der Turmmauer mit der westlichen Mauer des Konventhauses zu untersuchen (die Fundamente selbst konnten nicht erforscht werden); betrachtet werden konnte auch die Verbindung des Turms mit der Ostmauer der Hauptburg. Es ergab sich, dass die Ziegelsteinwände des Turms

12 Villem Raam, „Arhitektuur 13. sajandi teisest veerandist kuni 14. sajandi keskpaigani“, Eesti kunsti ajalugu I. Eesti kunst kõige varasemast ajast kuni 19. saj. keskpaigani, hrsg. von Irina Solomõkova u. a. (Tallinn: Kunst, 1975), 25. 13 Tomasz Torbus, Die Konventsburgen im Deutschordensland Preußen. Schriften des Bundesinstitut für ostdeutsche Kultur und Geschichte, Bd. 11 (München: R. Oldenbourg, 1998), 144ff.

14 Arvi Haak, ,,Arheoloogia andmed Viljandi ordulinnuse kesk- ja varauusaja kohta. Archaeological Data about the Viljandi Castle in the Medieval and Early Modern Period, Vijandi ordulinnus ja lossimäed läbi aja. The Teutonic Order's Castle Hills in
Viljandi: Viljandi Muuseum, 2015), 40.

15 Tuulse, Die Burgen in Estland und Lettland, 142 


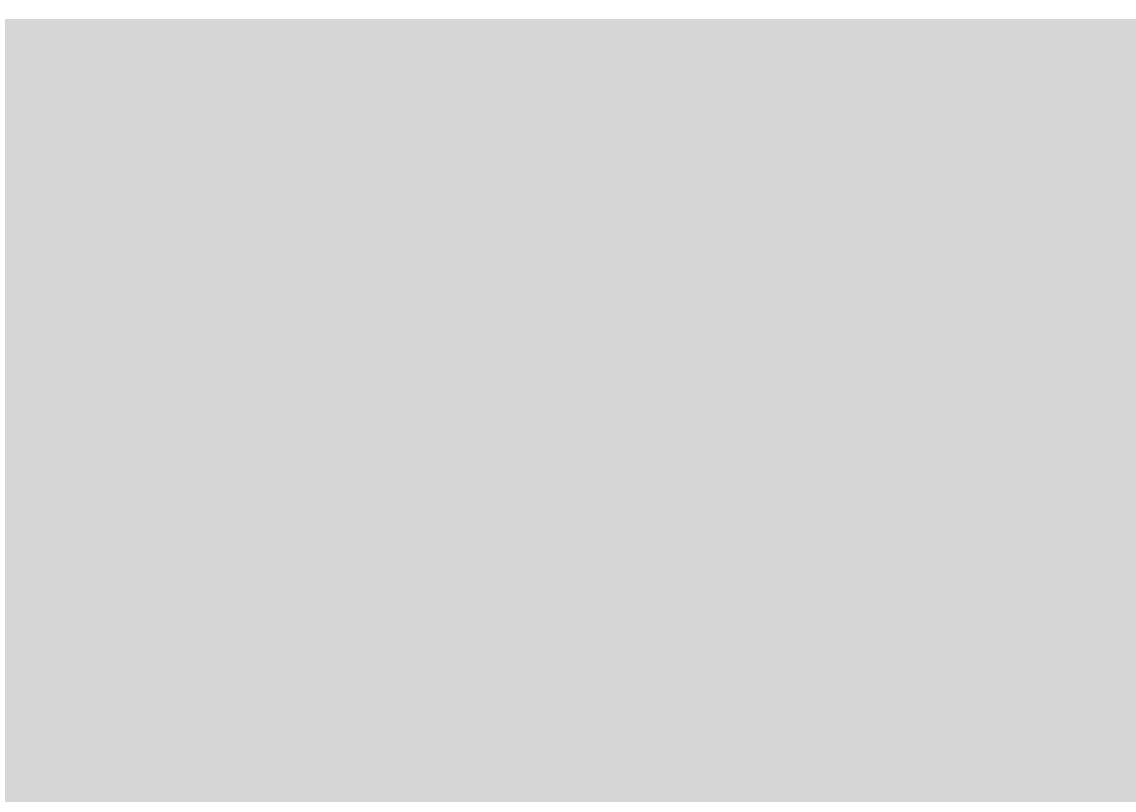

Abb. 3. Grundriss der Hauptburg in Fellin. Zeichnung von Elmo Raadik, ca. 1960.

ordentlich verfugt waren und dass die daran angrenzenden Mauern des Konventhauses nicht mit dem Turm verbunden waren, sondern gemauert, eben ohne Verbindung mit dem Turm. Daraus folgt, dass der Lange Hermann im Gegenteil als selbständiger Turm vor dem Konventhaus gebaut wurde. ${ }^{16}$ Der Turm verfügte im unteren Teil über einen tiefen, kellerartigen Raum - ein Zeichen das einem traditionellen Bergfried zueigen ist. Aufmerksamkeit erregt auch der Standort des Langen Hermann - er befand sich in der Nähe des Tors und kontrollierte den Zugang zum Burgplateau.

\section{BAUSKULPTUREN DER ORDENSBURG FELLIN}

Zu einer wahren Sensation der Ausgrabungen 1878-1879 wurde der Fund von zahlreichen Skulpturen als Baudetails. Hauptsächlich handelt es sich um Kapitelle und Säulenbasen ${ }^{17}$ Es handelt sich nach

16 Kalle Lange, Kaur Alttoa, „Die Turmburg in Estland“, Castella Maris Baltici 1, hrsg. von Knut Drake (Stockholm: Almquist \& Wiksell, 1993), 121.

17 Das gesamte Material gehört dem Viljandi Museum; ausgewählte Fundstücke sind im Zentrum für traditionelle Musik in Viljandi ausgestellt. den Terrakottaskulpturen der Dorpater (Tartuer) Johanniskirche um eine der wertvollsten Ansammlungen von mittelalterlichen Bauskulpturen in Estland. Die bis jetzt einzige Untersuchung der Kapitelle Fellins kommt aus dem Jahr 1938 von Armin Tuulse. ${ }^{18}$ Nach Auffassung dieses Autors stammten die gesamten Bauskulpturen aus dem Nordflügel des Konventhauses - aus der der Kapelle und dem Kapitelsaal. ${ }^{19}$ Offenbar ging er von Reinhold Gulekes Rekonstruktionszeichnungen aus. ${ }^{20}$ Tatsächlich ergibt sich aus den Angaben, welche die Ausgrabungen 1878/79 behandeln, dass derartige Details verstreut an mehreren Stellen auf dem Gebiet der Hauptburg aufgefunden wurden. So besteht also keine Grundlage dafür, das skulpturelle Dekor einzig mit den zwei genannten Räumen zu verbinden. Nach Einschätzung Armin Tuulses wurden sämtliche dieser Werke ungefähr im ersten Viertel des 14. Jahrhunderts von einem Meister geschnitten. ${ }^{21}$ Später hat auch Helmi Üprus die Leser mit diesen Standpunkten bekannt gemacht. ${ }^{22}$ Die in der Ordensburg Fellin aufgefundenen Bauskulpturen bedürften einer gründlicheren speziellen Abhandlung, doch dies stellt nicht das Ziel des vorliegenden Beitrags dar. Wir versuchen einzig, einige Momente zu markieren.

Auf mehreren Kapitellen befinden sich figurale Szenen. Deutlich ist die Geschichte vom Fuchs und dem Storch aus den Fabeln Äsops zu erkennen. Weiterhin sind auf den Kapitellen ausschlagende Ziegenböcke, ein Adler auf dem Rücken eines Schweins und irgendwelche langbeinigen Tiere dargestellt - das Kapitell ist schwer beschädigt und etwas Genaueres über diese Darstellungen kann nicht ausgesagt werden. Die genaue ikonografische Botschaft dieser Kapitelle konnte bis jetzt noch nicht entschlüsselt werden. In Einzelfällen verwiesen Forscher auf einzelne parallele Beispiele für die Motive. Beispielsweise trifft man auf die Geschichte von Fuchs und Storch im

18 Armin Tuulse, „Viljandi ordulossi kapiteelid“, Litterarum Societas Esthonica 1838-1938. Liber Saecularis II. Commentationes Litterarum Societatis Esthonicae XXX (Tartu: Õpetatud Eesti Selts, 1938), 755-769.

19 Ebenda, 757

20 Reinhold Guleke, Alt-Livland. Mittelalterliche Baudenkmäler Liv-, Est-, Kurlands und Oesels (Leipzig: K. F. Koehler, 1896), F. II. T. XV und XV.a.

21 Tuulse, „Viljandi ordulossi kapiteelid“, 761, 764

22 Helmi Üprus, Raidkivikunst Eestis XIII-XVII sajandini, hrsg. von Voldemar Vaga (Tallinn: Kunst, 1987), 46. 

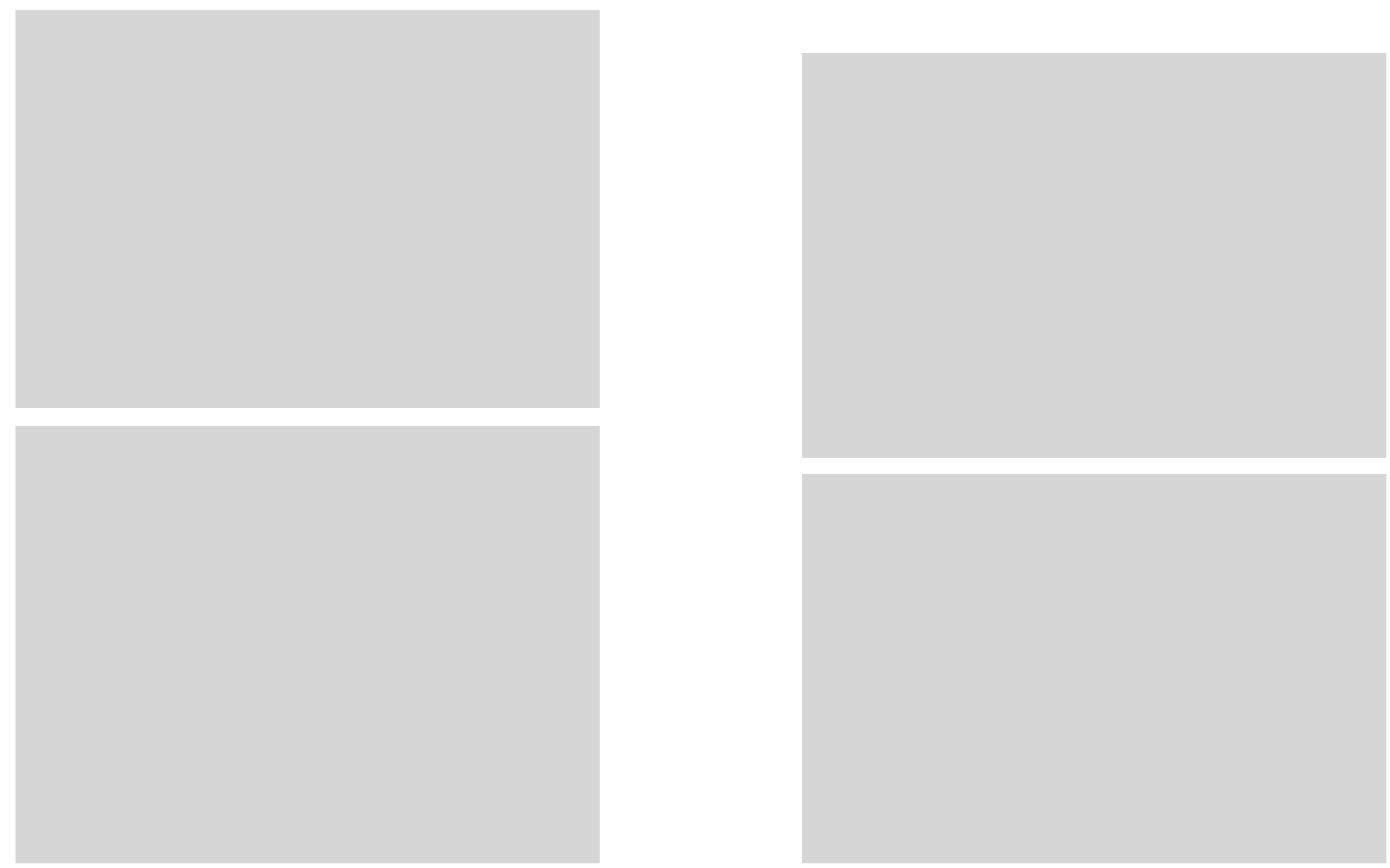

Abb. 6.-7. Kapitelle mit Figuren. Fotos: Kaur Alttoa. 


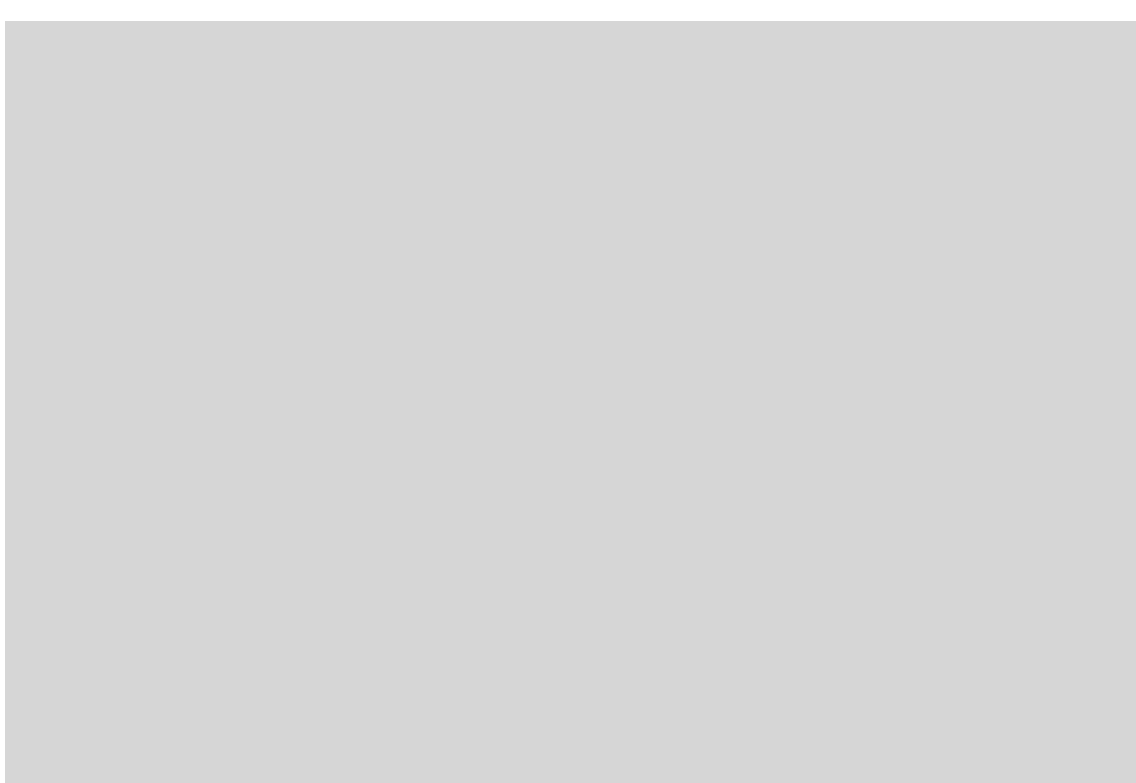

Abb. 8. Kapitell mit Weinblättern. Foto: Kaur Alttoa.

Lübecker Katharinenkloster ${ }^{23}$ und in der Paderborner Domkirche dort, in Paderborn, werden auf einem Kapitell auch ringende Bauern dargestellt. ${ }^{24}$ Es handelt sich um übereinstimmende Sujets oder Motive - natürlich können wir auf dieser Basis keine Folgerungen über einen gemeinsamen Meister oder die Genesis der Skulpturen treffen.

Am häufigsten findet sich auf den Kapitellen aber ein Pflanzendekor. Unter diesen unterscheidet sich eine kleine Gruppe von Arbeiten: ein Kapitell mit großen, naturalistischen Weinblättern und einige ähnlich modellierte Fragmente. ${ }^{25}$ Vom Stil her gehören sie bereits in die Hochgotik. Weiterhin findet sich in Estland noch ein Werk, bei dem sowohl die verwendeten Motive als auch die Handschrift der Verarbeitung den genannten Kapitellen aus Fellin sehr nahekommen. Es handelt sich um den Kämpfer des südlichen Portals der Kirche

23 Tuulse, ,Viljandi ordulossi kapiteelid“, 764.

24 Wilhelm Tack, ,Die Dombauten des 13. Jahrhunderts in Paderborn und Riga“, Westfälische Zeitschrift, 112 (1962), 238; Abb. 20-23.

25 Eines von diesen Kapitellen wurde bei den Ausgrabungen der Katharinen-Kapelle in Fellin gefunden (Kaur Alttoa, ,Die St. Katharinen-Kapelle in Fellin (Viljandi) “, Baltic Journal of Art History, 10 (2015), Abb. 21).

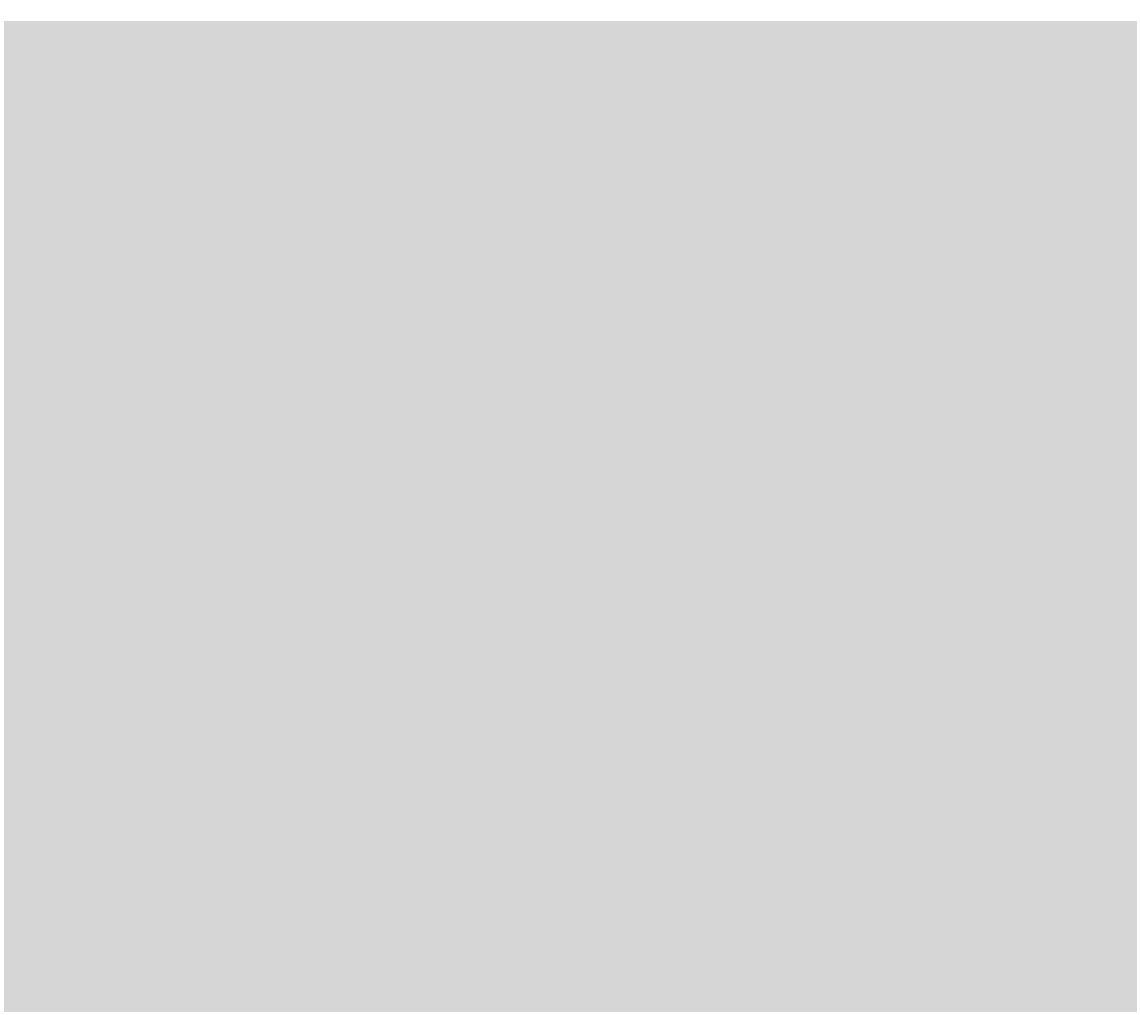

Abb. 9. Fragment des Kapitells. Foto: Kaur Alttoa.

in Torgel (Türi). Unter anderem hat der Meister in beiden Fällen als seinen eigenen Zugang ein Loch abgebildet, aus dem der Ast des Weins herauswächst. Sowohl in Fellin als auch in Torgel ist die Blattrippe ähnlich modelliert. Mit großer Sicherheit kann behauptet werden, dass es sich um das Werk ein und desselben Meisters handelt. Nach Einschätzung von Villem Raam ist die Kirche in Torgel und dessen Portal wahrscheinlich im letzten Viertel des 13. Jahrhunderts fertiggestellt worden, spätestens zum Jahr 1300. ${ }^{26}$ In diesen Zeitraum müsste auch das besprochene Kapitell aus Fellin zu verorten sein.

Auf der Rekonstruktion Reinhold Gulekes krönt das Kapitell mit dem Ast des Weins einen Pfeiler des Kapitelsaals. Dennoch stammt er offenbar aus dem Kreuzgang. Nämlich fand man bei den Ausgrabungen

26 Villem Raam, „Türi Martini kirik“, Eesti Arhitektuur 3. Harjumaa, Järvamaa, Raplamaa, Lä̈̈neVirumaa, Ida-Virumaa, Gesamthrsg. Villem Raam (Tallinn: Valgus, 1997), 91. 


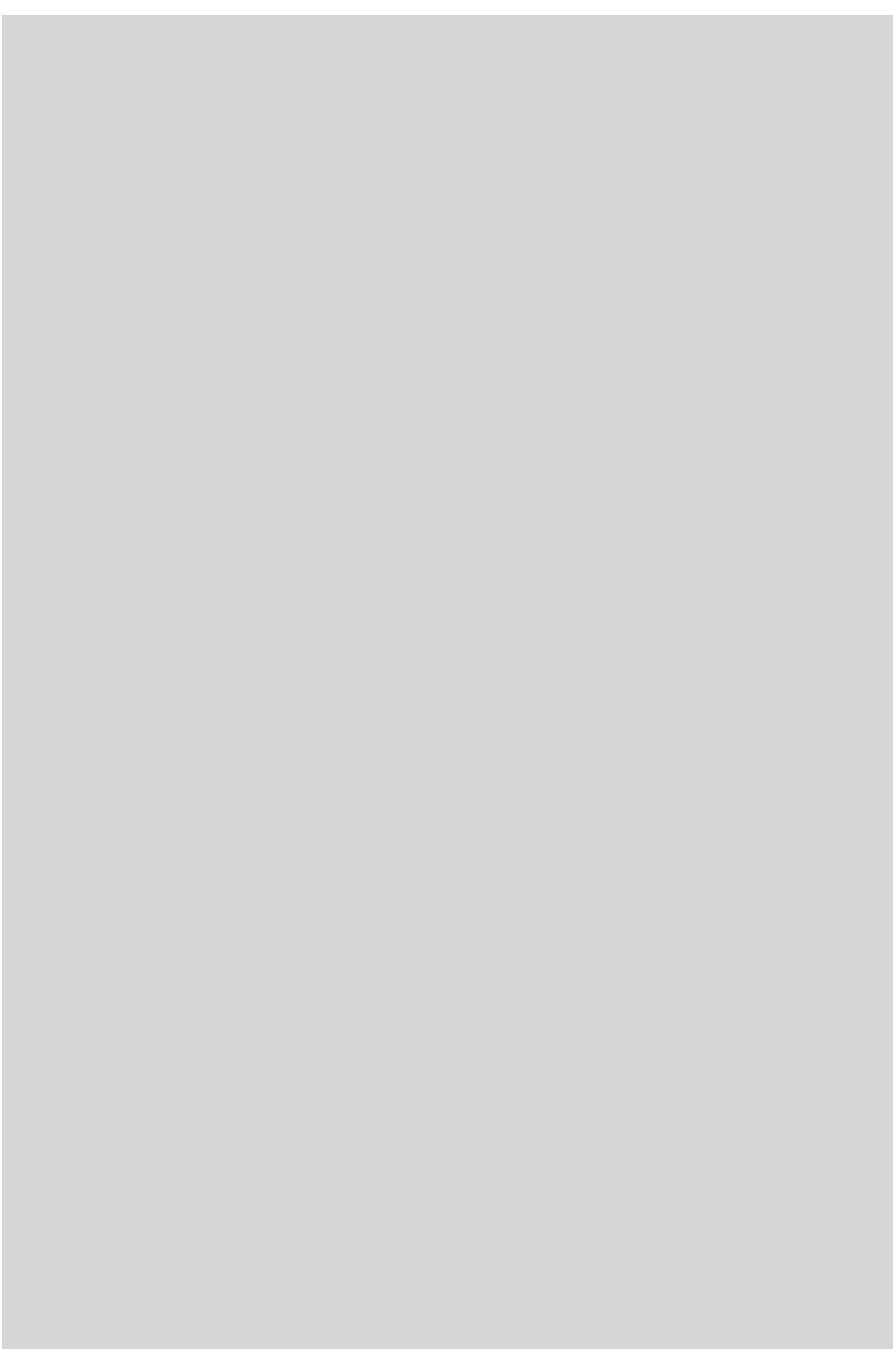

Abb. 10. Fragment des südlichen Portals der Kirche von Torgel. Foto: Kaur Alttoa.
1878/79 auf der West-, Süd- und auch der Ostseite des Hofes elf Überreste von Rundpfeilern des Kreuzgangs. ${ }^{27}$ Der Durchmesser des unteren Teils des Kapitells entspricht dem der Pfeilerschäfte des Kreuzgangs (54-56 $\mathrm{cm}$ ). Also wurde das Kapitell wahrscheinlich hergestellt, als es an der Tagesordnung war, den Kreuzgang zu erbauen. Es handelt sich dabei momentan um einen der wenigen Fixpunkte bei der Datierung des Konventhauses von Fellin - offensichtlich wurde dies zum größten Teil am Ende des 13. Jahrhunderts angefertigt.

Die überwiegende Mehrheit der Kapitelle ist aber erheblich archaischer. Hier sehen wir einzelne Gestaltungsmotive, welche der Romanik zueigen sind - beispielsweise Astragale. Dennoch besteht das charakteristischste Element in stilisierten Knospen in den Ecken des Kapitells. Es handelt sich um eine in der Frühgotik weit verbreitete Komposition, wofür sich ein eigener Terminus herausgebildet hat - Knospenkapitell.

Wann wurden aber die besprochenen Kapitelle geschaffen? Bislang ist es nicht geglückt in Alt-Livland oder den Nachbarländern Bauskulpturen zu entdecken, die vom Meister stammen könnten, der die Kapitelle in Fellin geschnitten hat. In Abwesenheit von besseren Ausgangsdaten müssen die altmodische vergleichende Methode und die Stilanalyse zu Hilfe genommen werden. Manche Folgerungen können bezüglich des Knospenkapitells gemacht werden. In der Domkirche in Riga findet man sie nahezu in Massen. ${ }^{28}$ Gemäß neueren Interpretationen wurden die jüngeren von ihnen in den fünfziger Jahren des 13. Jahrhunderts hergestellt. ${ }^{29}$ In Estland trifft man auf das Knospenmotiv beispielsweise in der Domkirche in Hapsal (Haapsalu) sowie in mehreren Parochialkirchen des Bistums Ösel-Wiek (Saare-Lääne) wie in Wolde (Valjala), Karmel (Kaarma), Pönal (Lääne-Nigula) ${ }^{30}$ und Goldenbeck (Kullamaa). Hier fällt die

27 Noch im Jahr 1980 waren zumindest zwei Überbleibsel dieser Pfeiler in situ erhalten.

28 Agnese Bergholde, Rīgas Doma viduslaiku arhitektūra un būvplastika eiropeisko analoğiju kontekstā (Rīga: Latvijas Māksalas akadēmijas Māksalas vēstures institūts, 2015), Plan S. 375.

29 Ebenda, 334.

30 Die Kirche in Pönal in Wiek ist bis jetzt auf das letzte Drittel des 13. Jahrhunderts datiert (Villem Raam, „Lääne-Nigula kirik“, Eesti Arhitektuur 2. Läänemaa, Saaremaa, Hiiumaa, Pärnumaa, Viljandimaa, Gesamthrsg. Villem Raam (Tallinn: Valgus, 1996), 29). In den neunziger Jahren des 20. Jahrhunderts ergab sich, dass sich im Chorraum der Kirche in der Ostwand ein Doppelfenster befunden hatte. Ein solches in Karmel auf Ösel (Saaremaa) sowie in den Kirchen von Hanhel (Hanila) und St. Mararethen (Karuse) in Wiek. Nach den sechziger Jahren des 13. Jahrhunderts erscheint eine solche Fensterkomposition nicht mehr und offensichtlich muss auch die Datierung der Kirche in Pönal auf eine etwas frühere Zeit verschoben werden. 
Verbreitung des Baus von Knospenkapitellen vor allem in die Jahre 1250-1260, in Einzelfällen können sie als Ausnahme auch später angetroffen werden.

In der Ordensburg Fellin befanden sich nebeneinander Bauskulpturen mit Merkmalen sowohl der Romanik, der Frühgotik als auch der Hochgotik. Natürlich kann es hier verschiedene Gründe geben: beispielsweise arbeiteten Meister verschiedener Generationen und genutzt wurden unterschiedliche Notizbücher. Auf jeden Fall kann sich der Verfasser nicht mit der Interpretation einverstanden erklären, dass sämtliche Kapitelle von einem Meister geschaffen wurden. Ebenfalls erscheint es als sehr fragwürdig, dass diese alle innerhalb desselben Zeitraums fertiggestellt wurden. Wahrscheinlich trennen die archaischen Kapitelle der Romanik und die der Hochgotik etwa dreißig Jahre. Daraus ergibt sich aber eine wichtige Folgerung: offenbar wurde ein Teil der Kapitelle zu einer Zeit hergestellt, als sich das Konventhaus in Preußen noch nicht herausgebildet hatte. Wie bereits erwähnt, verläuft hier die Zeitgrenze in den achtziger Jahren des 13. Jahrhunderts. In dem Fall, dass wir nicht behaupten wollen, dass dieser Burgtyp in Fellin entwickelt wurde, ${ }^{31}$ muss demnach gefolgert werden, dass ein ziemlich großer Teil des steinhauerischen Dekors in der Hauptburg Fellins ein zweites Mal genutzt wurde. Und anfangs gehörte es wohl zu irgendeinem anderen Gebäude.

Ich habe zu meiner Zeit auf die Möglichkeit verwiesen, dass zwischenzeitlich der Nordflügel des Konventhauses vorläufig ein eigenständiges Gebäude war, das im Folgenden in das zu errichtende Konventhaus integriert wurde. ${ }^{32}$ Spätere archäologische Untersuchungen haben aber gezeigt, dass offensichtlich sämtliche vier Flügel des Konventhauses nach einem Grundriss gebaut wurden ${ }^{33}$ und diese Version also ignoriert werden sollte. Danach entstand

31 Übrigens ist August Westrén-Doll im Jahr 1929 zu diesem Kuriosum gekommen. Er gehörte zu jener Generation von Forschern, die dazu neigte, die Grundlegung-, Bau- und Fertigstellungszeit eines Gebäudes gleichzusetzen, und nicht berücksichtigte, dass ein größeres Gebäude aus Teilen bestehen könnte, die zu verschiedenen Zeiten errichtet worden waren. Der Gedankengang dieses Autors war einfach: Die Steinburg in Fellin wurde 1224 gebaut (laut Information der Chronik begann man gerade dann mit der Errichtung der Steinburg!), die Marienburg (Malbork) wurde aber erst viel später gebaut (Grundlegung 1279 - K. A.). Folglich war Fellin also das Vorbild für das Konventhaus in Marienburg (August WestrénDoll, , ,Burg und Stadt Fell in zu polnischer Zeit , Sitzungsberichte der Gelehrten Estnischen Gesellscha 1928 (Tartu-Dorpat: Gelehrte Estnische Gesellschaft zu Dorpat, 1929), 72-73).

32 Kaur Alttoa, „Das Konventhaus in Estland“, Castella Maris Baltici 1, hrsg. von Knut Drake (Stockholm: Almquist \& Wiksell, 1993), 12.

33 Arvi Haak, Anu Vaba, Heiki Valk, ,2001. aasta arheoloogilised uuringud Viljandis“, Viljand Munseumi aastaraamat 2001 (Viljandi: Viljandi Muuseum, 2002), 36-37. eine neue Hypothese: vor dem Konventhaus befand sich auf dem Territorium der Ordensburg Fellin irgendein anderes früheres Repräsentationsgebäude - ein Palas, über dessen Standort wir nichts wissen. ${ }^{34}$ Unter Berücksichtigung des Reichtums des steinhauerischen Dekors kann ohne Zweifel behauptet werden, dass vor dem Bau der Kirche in Karris (Karja) dieses Gebäude nach der Domkirche in Riga das repräsentativste Gebäude in ganz Alt-Livland darstellte. Das Konventhaus in Fellin und das es umgebende Gebiet ist bei weitem nicht archäologisch durchforscht. Dennoch erscheint es als schwer, einen Ort zu finden, wo sich ein derartig großangelegtes Gebäude befunden haben könnte.

\section{ZWILLINGSSÄULCHEN}

Es gilt, den Ursprungsort der archaischen Kapitelle in Fellin zu suchen. Dabei muss gesondert auf eine Gruppe von behauenen Steinen verwiesen werden. Denn es wurden in der Ordensburg zahlreiche Zwillingskapitelle aufgefunden, dazu zählen kleine Säulchen (Durchmesser 11,5-13,5 cm), Schäfte und entsprechende Basen - die meisten von ihnen verfügen über Eckblätter. ${ }^{35}$ Ein Teil der Säulchen war freistehend, ein anderer aber grenzte an die Wand. Laut Reinhold Gulekes Rekonstruktion ${ }^{36}$ handelte es sich um Fenstersäulen, die aus dem Kapitelsaal und der Kapelle stammten. Auf demselben Standpunkt beharrt auch Armin Tuulse. ${ }^{37}$ Auf Gulekes Zeichnungen ist der obere Teil der Fenster als Dreibogen dargestellt. Eine derartige Form dachte sich der Architekt Guleke nicht selbst aus: unter den Fundstücken der Burg befinden sich mehrere Baudetails aus Kalkstein, aus denen sich ein Sturz in entsprechender Form ableiten lässt. ${ }^{38}$

34 Kaur Alttoa, „Viljandi ordulinnuse arhitektuur. On the Architecture of Viljandi Castle“, Viljandi ordulinnus ja lossimäed läbi aja. The Teutonic Order's Castle Hills in Viljandi Through Time. Viljand Muuseumi toimetised V (Viljandi: Viljandi Muuseum, 2015), 107-108. 35 In der estnischen architektonischen Literatur wird das Eckblatt als zur Romanik zugehörig bezeichne
(Tuulse, ,Viljandi ordulossi kapiteelid“, 763; Eesti arhitektuur. Oskussõnastik (Tallinn: Valgus, 1993), 19). Tatsächlich wird dieses Motiv zahlreich beispielsweise in der Kathedralengotik der Île-de-France angetroffen.

36 Siehe Fn. 20

37 Tuulse, „Viljandi ordulossi kapiteelid“ 759

38 Diese Details sind etwa $30 \mathrm{~cm}$ lang - tatsächlich stützte sich der Sturz auf eine Ziegelsteinmauer von der Stärke eines Ziegelsteins. 

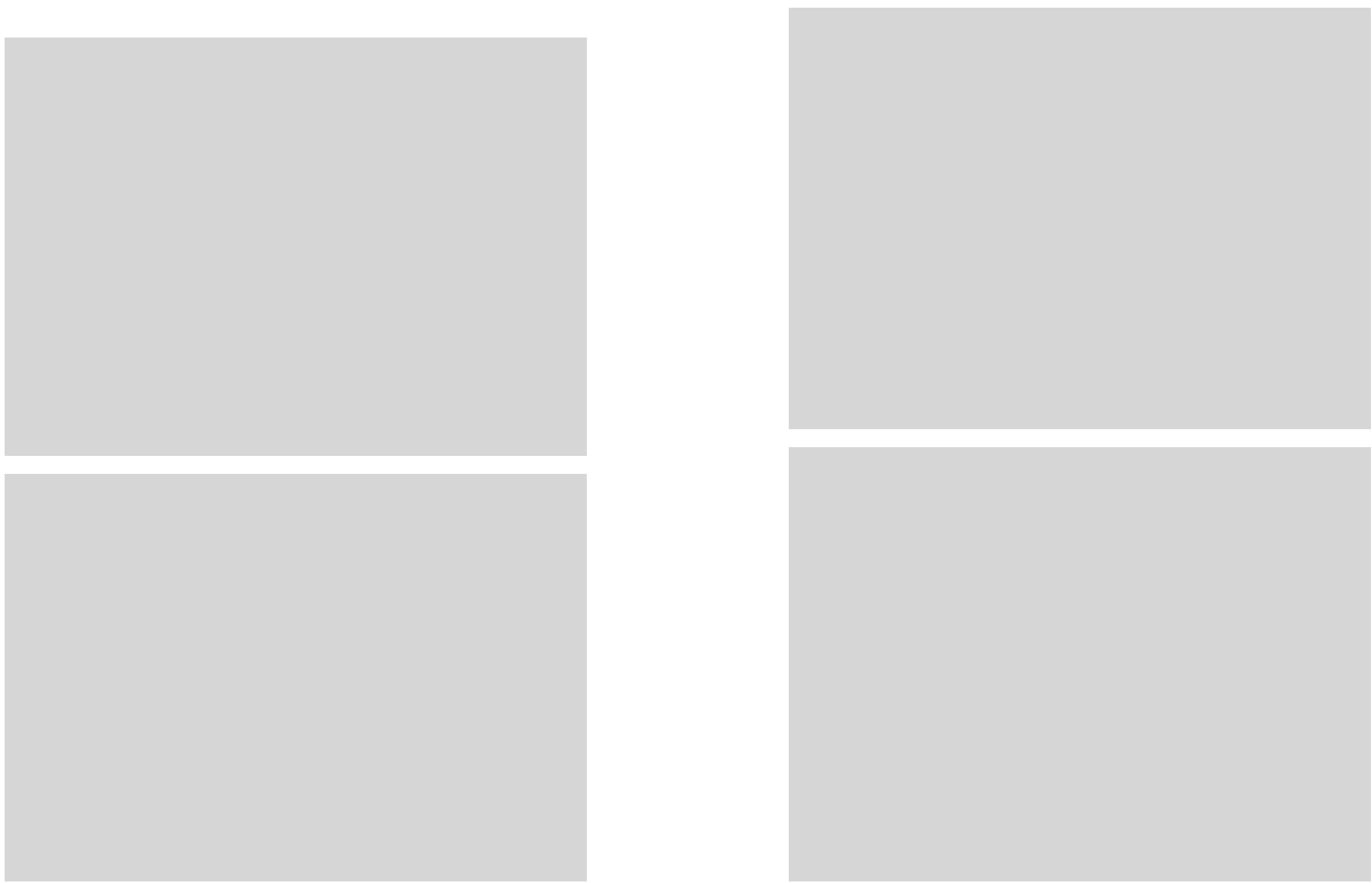

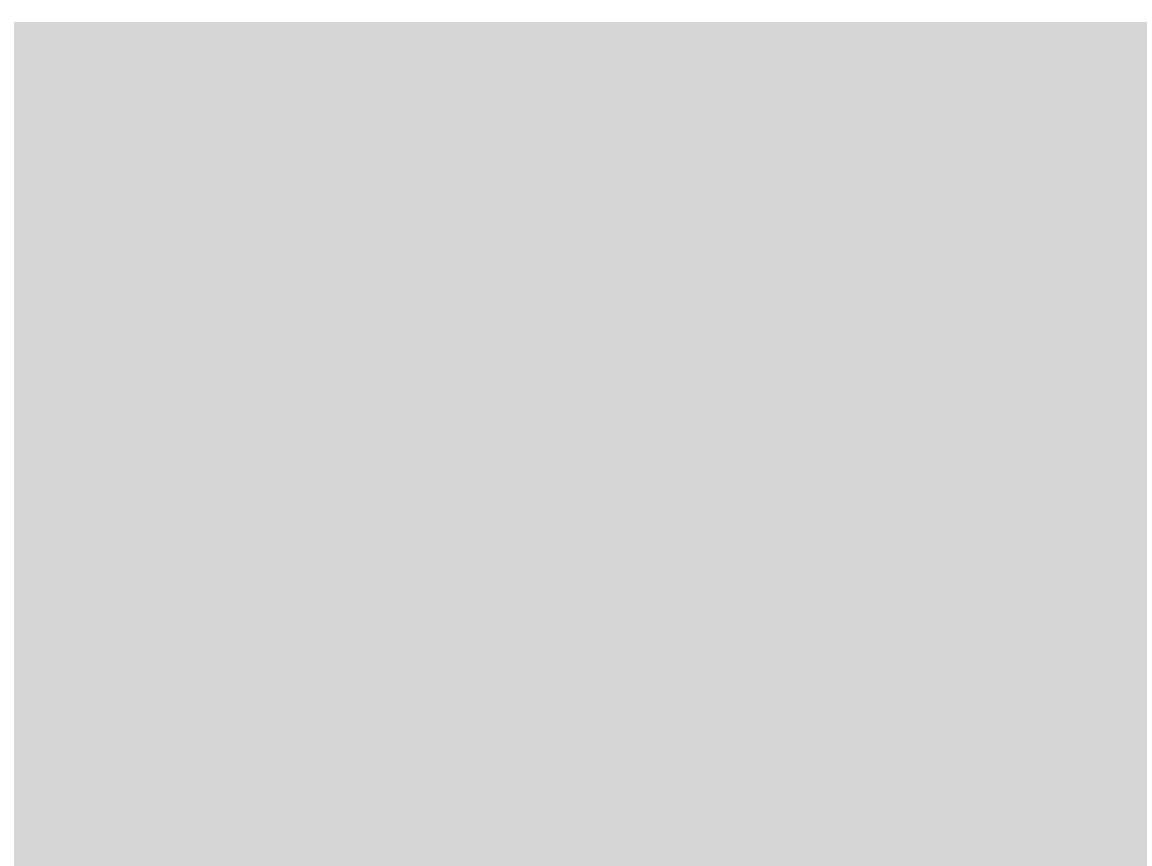

Abb. 13.-14. Kapitelle der Zwillingssäulchen. Fotos: Kaur Alttoa. 
Dennoch kann im vorliegenden Fall diese Rekonstruktion aus mehreren Gründen nicht akzeptiert werden. In Estland - oder wenigstens in Reval (Tallinn) - wurden tatsächlich Kompositionen verwendet, bei denen sich bei einem Zwillingsfenster mit Segmentbogen oder horizontalem Sturz in der Mitte sich ein verziertes Säulchen befindet. Eine derartige Gestaltung nahm ihren Anfang aber im 15. Jahrhundert und wurde im nachfolgenden Zeitalter der Renaissance besonders populär. ${ }^{39}$ Doch in früheren Jahrhunderten scheint eine derartige Gestaltung unbekannt gewesen zu sein.

Was aber die Hauptsache ist, bei einem behauenen Steinrahmen für ein verglastes Fenster musste sich unbedingt ein Falz für den Glasrahmen befinden, gewöhnlich aus Eisen. Bei den entsprechenden Baudetails, die im Museum Viljandi aufbewahrt werden, fehlt dieser aber. Damit gehören diese Teile nicht zu einem verglasten Fenster. Offenbar stammen sie aus irgendeiner offenen Galerie oder aber, was noch viel glaubwürdiger ist, aus dem Kreuzgang.

Eins muss sofort präzisiert werden, dass es sich auf gar keinen Fall um den Kreuzgang des Konventhauses in Fellin handeln kann. Dort befanden sich auf der Hofseite solide Rundpfeiler, zwischen denen keinerlei ergänzende kleinere Arkaden eingebaut werden können. Die besprochenen Kapitelle und Säulchen mit Füßen gehörten im Gegenteil zu einer Galerie, deren Gestaltung an den Kreuzgang der Domkirche zu Riga erinnerte. Es geht natürlich einzig um die allgemeine Komposition, nicht um die Details. Somit gehörte zum gesuchten Gebäude auch eine offene Galerie oder ein Kreuzgang.

In dieser Situation tritt unvermeidlich die Frage auf, ob die gesamten Bauskulpturen anfänglich die Ordensburg Fellin verschönerten? Zwischendurch wurden sie vielleicht von anderswo hergebracht? Auf den ersten Blick erscheint eine solche Vermutung als äußerst fragwürdig. Dies bedeutete, dass es im 13. Jahrhundert in Alt-Livland irgendwo ein besonders reich gestaltetes Gebäude gab, welches bereits weniger als ein halbes Jahrhundert nach der Fertigstellung abgerissen wurde und Teile verteilt und wiederverwendet wurden Und dieses Gebäude müsste entweder über eine Galerie oder einen
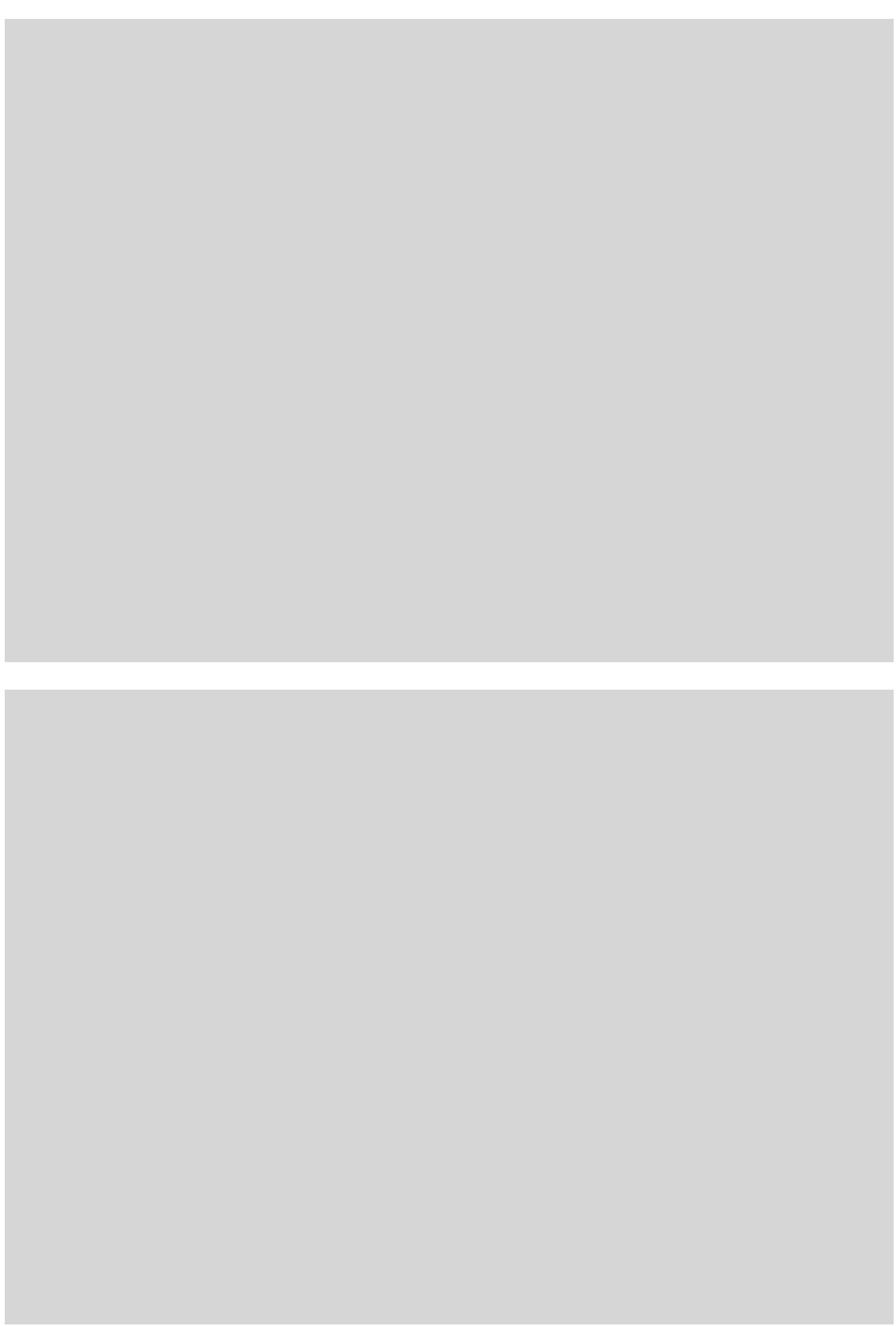

Abb. 15.-16. Die Basen der Zwillingssäulchen. Foto: Kaur Alttoa. 


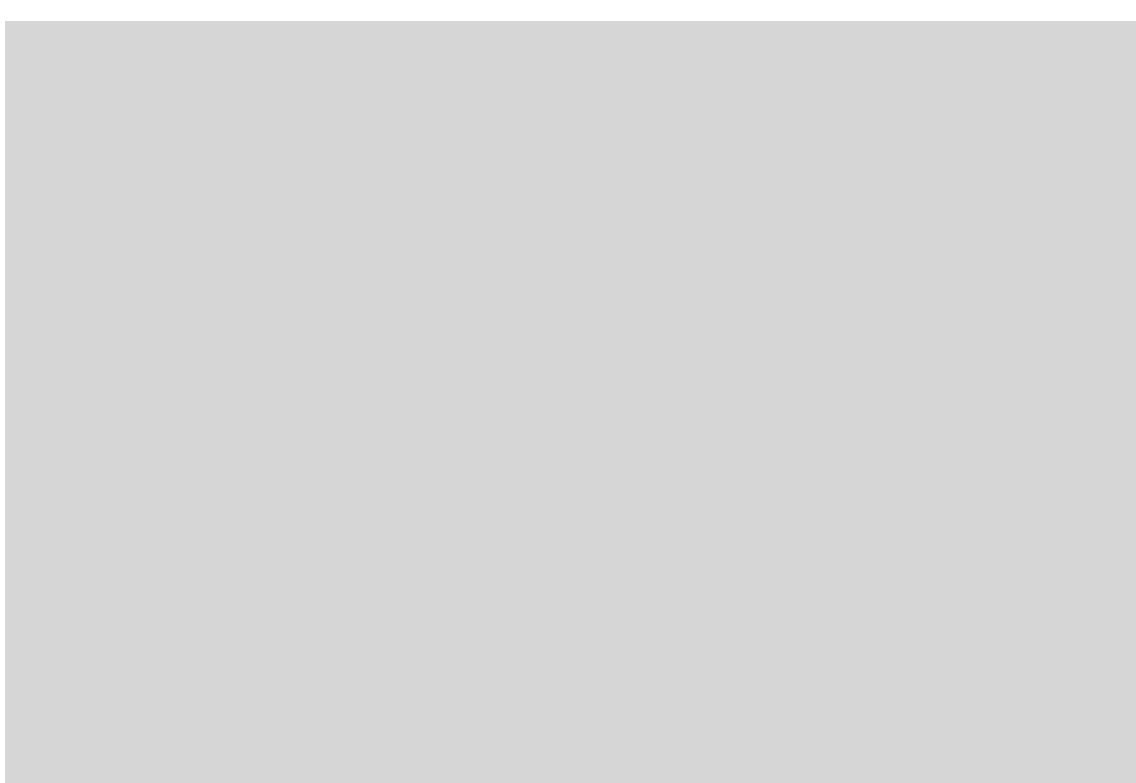

Abb. 17. Kapitelsaal und Kapelle. Rekonstruktion von Reinhold Guleke.

Kreuzgang verfügen. Derartige Gebäude konnte es damals in AltLivland nur ausgesprochen wenige geben. Tatsächlich ist ein solcher Fall nicht weit entfernt von Fellin wirklich vorhanden - es handelt sich nämlich um:

\section{DIE DOMKIRCHE VON ALT-PERNAU ${ }^{40}$}

In der Anfangszeit des Bistums Ösel-Wiek hat das Zentrum dieses Bistums mehrfach seinen Standort gewechselt. Am Anfang saß der Bischof in Leal (Lihula). Aus dem Jahr 1238 stammt eine Urkunde, worin unter anderem der Plan erwähnt wird, eine Domkirche zu errichten. Wohl wurde eine Klausel hinzugefügt, dass in den

40 Theoretisch müsste noch ein zweites Gebäude berücksichtigt werden, das Zisterzienserkloster in Dünamünde (Daugavgrīva). Am Anfang des 14. Jahrhunderts wurde dies dem Livländischen Orden verkauft, der anstelle des Klosters eine Burg errichtete. Diese Vermutung unterstützt der Umstand, dass sowohl Dünamünde als auch Fellin dem Orden gehörten, im Fall von Alt-Pernau handelte es sich abe um einen ganz anderen Machthaber. Andererseits, Dünamünde ging im Jahr 1305 an den Orden über Papst Johannes XXII. bestätigte den Verkauf jedoch erst 1319 (über den Verkauf des Klosters: Lore Poelchau, Geschichte des Zisterzienserklosters Dünamünde bei Riga (1205-1305). Sonderdruck aus der Reihe „Studien und Mitteilungen zur Geschichte des Benediktinerordens und seiner Zweige“ (St bereits fertiggestellt und es bestand kein Bedarf mehr nach irgendwelchen alten Kapitellen. folgenden drei Jahren das Einkommen noch derartig wachsen müsse, um eine Burg zu bauen. ${ }^{41}$ Aus dem Jahr 1251 stammt aber eine Urkunde, in welcher der Bischof von Ösel-Wiek, Heinrich, bekannt gibt, dass eine Domkirche in Alt-Pernau (Perona) eingerichtet wurde. ${ }^{42}$ Mehrere Forscher haben behauptet, dass zuerst geplant war, eine Domkirche in Leal zu bauen. Dennoch erscheint es als glaubwürdig, dass bereits bei der Planung einer Kathedrale 1238 vorgesehen war dass sie sich in Alt-Pernau befinden sollte. ${ }^{43}$ Am 23. August 1253 bestätigt der Erzbischof die Errichtung der Kathedrale ${ }^{44}-$ sicherlich war zu diesem Zeitpunkt wenigstens die Domkirche fertiggestellt. Die Litauer zerstörten die Kirche 1263 und anschließend wurde Hapsal zum Zentrum des Bistums.

Die Urkunde von 1251 ist sehr informationsreich. Unter anderem stellt Bischof Heinrich dort auch Regeln vor, nach denen die Mitglieder des Domkapitels in Klausur zu leben haben, gesondert werden sowohl der Kapitelsaal, das Refektorium als auch das Dormitorium erwähnt. Detailliert werden auch die Strafen aufgezählt, die für eine Verletzung der Regeln des gemeinsamen Lebens den Domherren verhängt werden. Eine solche Konkretheit erlaubt es zu vermuten, dass nicht die Rede über eine in ferner Zukunft einzurichtende Institution war, sondern über eine Einrichtung, die in naher Zukunft aktiv sein wird. Damit müssten die Bauwerke der Klausur sich 1251 bereits in der Nähe des Abschlusses befunden haben.

Das einzige Planschema des bischöflichen Gebäudekomplexes in Alt-Pernau hat Carl Russwurm vorgelegt, der zu seiner Zeit von Einwohnern der Gegend Angaben über Mauerreste gesammelt hatte und auf dieser Grundlage einen Plan der Kirche und der KlausurAnlage erstellte. ${ }^{45}$ Vor Ort erhielt er einige wenige Informationen $\mathrm{zu}$

41 Liv-, Esth- und Curländisches Urkundenbuch nebst Regesten (weiterhin: LUB), Bd. 1, hrsg. von Friedrich Georg von Bunge (Reval: Kluge und Ströhm, 1853), Nr. 156; LUB, Bd. 3, hrsg. von Friedrich Georg von Bunge (Reval: Kluge und Ströhm, 1857), Nr. 156.

42 Carl Russwurm, Nachrichten über Alt-Pernau (Reval: Lindfors' Erben, 1880), 32-35; Pärnu linna ajaloo allikad 13 -16, sajandini, I. Teil hrsg von Inna Põltsam, Aldur Vunk (Pärnu: Pärnu linnavalitsus, 2001), 17-18. Jaanika Päll und Kaspar Kolk halfen, die Urkunden zu interpretieren, der Autor bedankt sich herzlich dafür

43 Siehe ausführlicher: Kaur Alttoa, „Kui piiskop polnud veel Haapsallu jõudnud“, Saare-Lääne piiskopiriik ja Haapsalu piiskopilinnus. Läänemaa Muuseumi toimetised XVI (Haapsalu: Haapsalu ja

44 LUB, Bd. 6, hrsg. von Friedrich Georg von Bunge (Riga: N. Kymmel, 1873), 23, 2734

45 Russwurm, Nachrichten über Alt-Pernau, T. 1. 


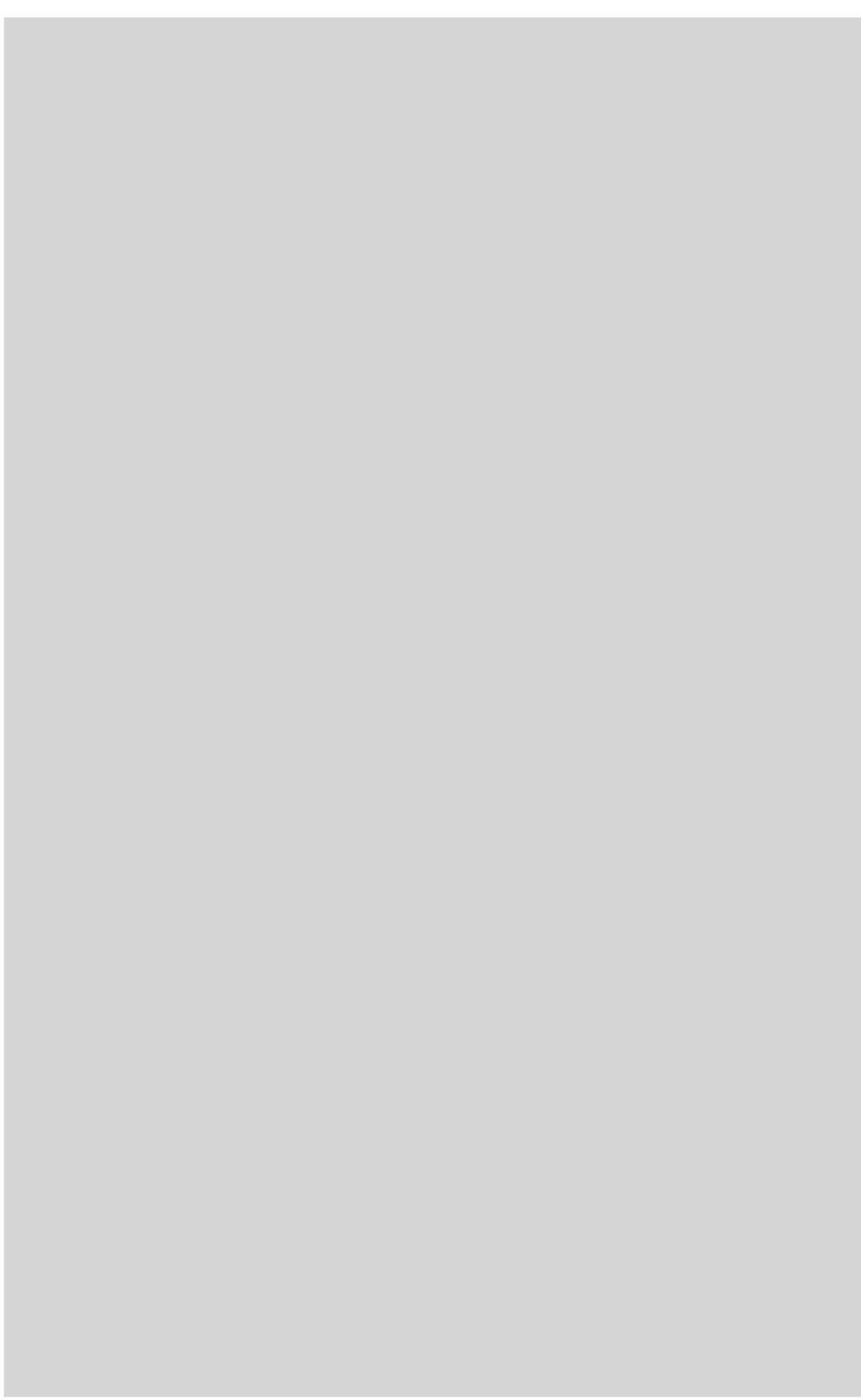

Abb. 18. Stürze aus Kalkstein. Foto: Kaur Alttoa.

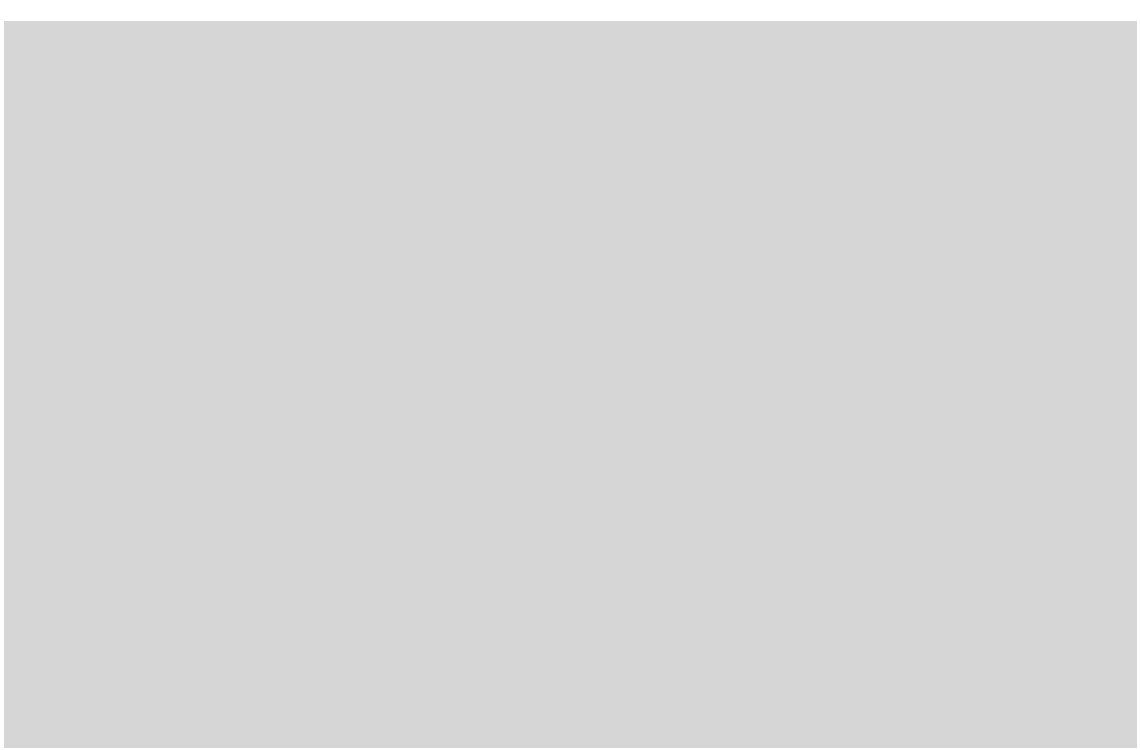

Abb. 19. Fensterstürze. Rekonstruktion von Reinhold Guleke.

den Mauern der Domkirche, ${ }^{46}$ doch bei deren Darstellung ist er nicht folgerichtig. ${ }^{47}$ Bezüglich der Klausur hatte er aber keinerlei Angaben von Beobachtungen. Auf der Zeichnung sah die Anlage ähnlich aus wie die in Hapsal - diese bestand aus Flügeln, die im rechten Winkel zueinanderstanden. Den südlichen Flügel belegte die Domkirche. Wohl besteht die Vermutung, dass Russwurm beim Zeichnen des Schemas den Grundriss der Burg Hapsal vor sich liegen hatte, ${ }^{48}$ dessen Angaben er wegen des Fehlens besserer Informationen auf Alt-Pernau projizierte. Wohl ist aber bezüglich Alt-Pernau deutlich, dass eine Klausur mit einem derartigen Raumprogramm unbedingt auch über einen Kreuzgang verfügen musste. So manches Mal wurde mit dem Bau des Kreuzgangs erst dann begonnen, wenn das daran angrenzende Hauptgebäude bereits fertiggestellt war. Bei einem schlechten Baugrund

46 Russwurm, Nachrichten über Alt-Pernau, 16

47 Auf Russwurms Zeichnung stehen die Proportionen der Kirche im Verhältnis 1:2 - damit könnte an dieser Stelle gefolgert werden, dass es sich um eine zweijochige Kirche gehandelt hatte. Zugleich verkündet er aber, dass die Kirche die Ausmaße von $108 \times 49$ englische Fuß hatte (Russwurm, Nachrichten über

48 Dieser Forscher hatte sich auch mit der Burg zu Hapsal beschäftigt. Carl Russwurm, Das Schloß zu Hapsal in der Vergangenheit und Gegenwart (Reval: Franz Kluge, 1877). 


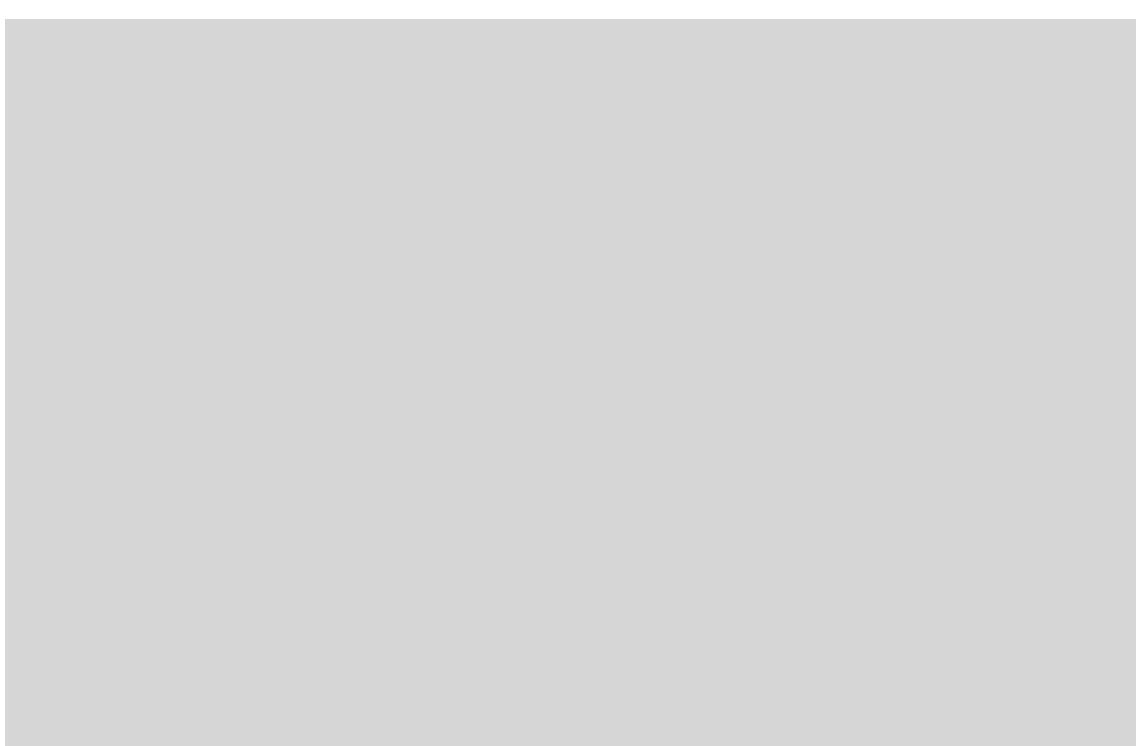

Abb. 20. Der Kreuzgang der Domkirche zu Riga. Foto: Kaur Alttoa.

wurde dies sogar empfohlen. Nämlich lastet auf der Wand zur Hofseite des Kreuzgangs eine ungleich kleinere Last als auf derjenigen zur Gebäudeseite. Bei einem gleichzeitigen Bauen kann dies zu einem ungleichen Einsinken der Wände führen, was besonders gefährlich für die Gewölbe ist. Dieses Risiko verringert sich, wenn mit dem Ausbau des Kreuzgangs erst begonnen wird, wenn sich die Mauern des Hauptgebäudes schon stabilisiert und gesetzt haben.

Damit mag behauptet werden, dass der Kreuzgang der Domkirche zu Alt-Pernau irgendwann in den fünfziger Jahren des 13. Jahrhunderts erbaut worden war, es ist sogar nicht ausgeschlossen, dass dies erst in der zweiten Hälfte des Jahrzehnts erfolgte.

Es fehlt jegliche Information darüber, wie die Domkirche und die Klausurräume in Alt-Pernau gestaltet waren. Zur selben Zeit darf eine besondere Linie der Sakralarchitektur Ösel-Wieks nicht vergessen werden - beginnend mit den ältesten Kirchen befanden sich auch Bauskulpturen an einer hervorgehobenen Stelle (die Parochialkirchen in Wolde und Karmel). Es wäre eigenartig vorauszusetzen, dass die Hauptkirche des Bistums ärmer gestaltet war als zur selben Zeit errichtete Dorfkirchen. Auf jeden Fall erscheint es als sehr wahrscheinlich zu vermuten, dass sich in der Kathedrale von Alt-Pernau und in der daran grenzenden Klausur auch Bauskulpturen befanden.

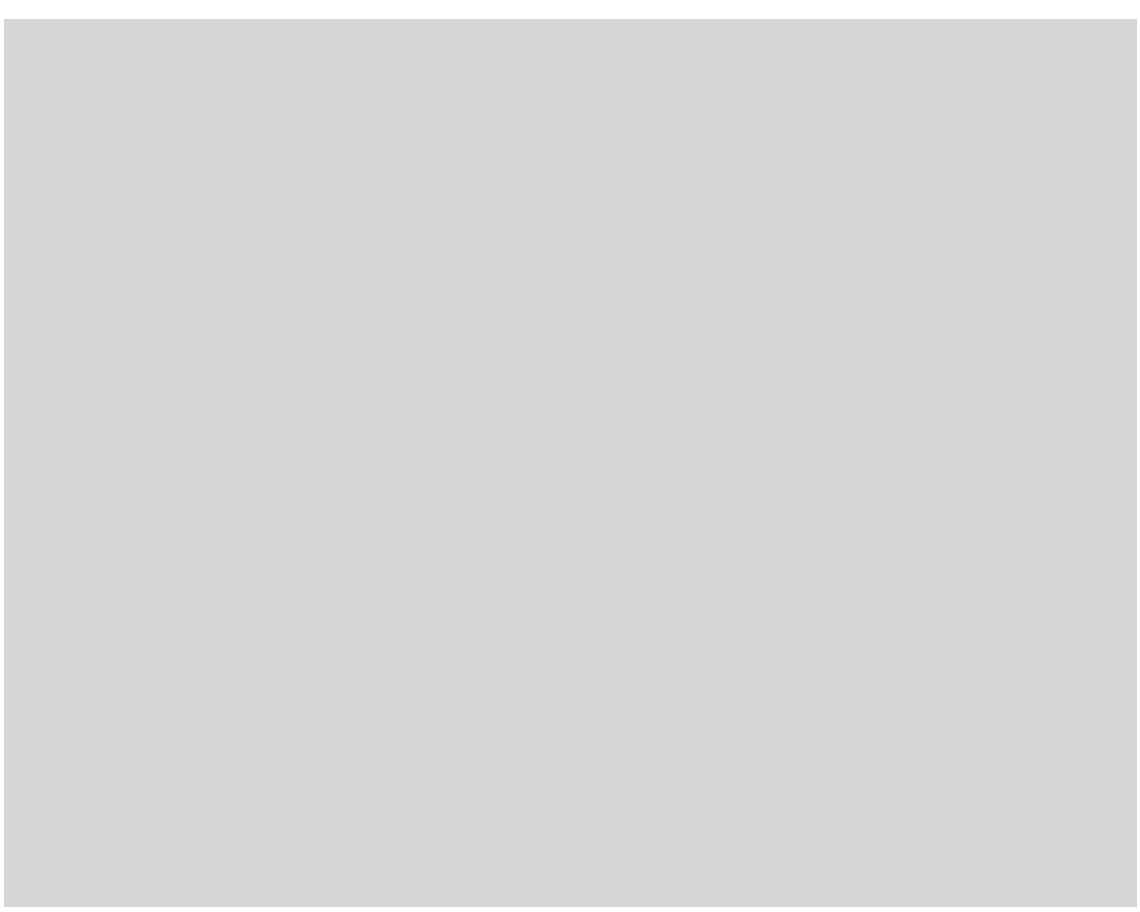

Abb. 21. Die Domkirche in Alt-Pernau und ihre Umgebung. Rekonstruktion von Carl Russwurm.

Die Existenz der Domkirche von Alt-Pernau blieb sehr kurzfristig, wie oben erwähnt, wurde sie im Jahr 1263 zerstört. Natürlich muss nicht vermutet werden, dass im Verlauf eines Raubzugs ein aus Stein errichtetes Gotteshaus dem Erdboden gleichgemacht wird Anschließend wurde die ehemalige Domkirche als Pfarrkirche St. Thoma wiederaufgebaut. Es ist unbekannt, wann dies erfolgte erstmals erscheint die Kirche in schriftlichen Quellen im Jahr $1363 .{ }^{49}$ Es erscheint als eindeutig, dass eine Pfarrkirche kein Klausurgebäude braucht. Damit erwies sich auch der Kreuzgang als nutzlos.

\section{FAZIT}

In Anbetracht der außergewöhnlich reichen Bauplastik der Burg Fellin sollte mit der Möglichkeit gerechnet werden, dass ein Teil davon aus der 1263 zerstörten Domkirche in Alt-Pernau stammte.

49 LUB, Bd. 6, Reg. 1178 k; 1223 b. 
Gleichzeitig bestehen keine Zweifel, dass sich in der Bauhütte, die das Konventhaus in Fellin errichtet hatte, wenigstens ein Steinmetz hoher Qualifikation befand - ein Meister, der auch das südliche Portal der Kirche zu Torgel gehauen hatte.

Aus dem Viljandi-See entspringt der Fluss Raudna, der in Verbindung mit dem Fluss Pärnu steht. Damit bestand zwischen Pernau und Fellin zumindest ein ordentlicher Winterweg. Auf jeden Fall bleibt die Frage bestehen, ob irgendwann am Ende des 13. Jahrhunderts dort entlang die Stücke von Bauskulpturen transportiert wurden, mit denen in Alt-Pernau nichts mehr anzufangen war. In Fellin aber erfolgte zur selben Zeit ein großangelegter Burgbau.

Der vorliegende Beitrag ist wie eine Gleichung, die hauptsächlich aus unbekannten Größen besteht. Vielleicht könnte die Frage durch umfangreiche bauarchäologische Ausgrabungen am Standort der Domkirche in Alt-Pernau erhellt werden. Doch dass diese erfolgen, ist zumindest in der nahen Zukunft ausgesprochen unwahrscheinlich.

KAUR Alt TOA: The column capitals of the Order castle in Viljandi - Were they second-hand goods from Old Pärnu? KEYWORdS: Viljandi Castle; Old Pärnu Cathedral; medieval STONE CARVING; CLOISTER

\section{SUMMARY}

The rediscovery of the Viljandi Castle, which was totally destroyed during the Great Northern War, occurred between 1878 and 1879, when extensive excavations were conducted under the direction and guidance of Theodor Schiemann (1847-1921). The real sensation revealed by the excavations at the time was the discovery of numerous carved construction details. Most of them were column capitals or bases. These have been attributed to the main castle, which was a convent building typical of the Teutonic Order. This type of castle was developed in Prussia between ca 1280 and 1300, and its "classic" form spread between 1300 and 1330, during approximately the same half century as the Viljandi convent building was built.
Some of the Viljandi column capitals have figural decorations and oak leaves are most common. There is also a large capital with naturalistic grape leaves, which comes from a cloister and was completed in the late $13^{\text {th }}$ century. Apparently, the convent building was being constructed at that time.

However, most of the capitals are much more archaic. Some of the motifs are typical of the Romanesque style. But the most common are the so-called "bud capitals" typical of the Early Gothic style. Basically such decorations became popular in Old Livonia in the 1250s and 1260 s. In any case, it is clear that a large number of the capitals were carved when the convent-type castles had yet to develop.

In the past, I have alluded to the possibility that there was a large richly decorated structure in Viljandi which was demolished to build the convent building. However, this is extremely unlikely. Although an archaeological study has not been made of the entire area of Viljandi's main castle, it is hard to identify a place where such a large-scale building could have existed.

Therefore, the possibility should be considered that some of the caitals were brought from elsewhere. We should also turn our attention to the fact that there are numerous capitals and bases for paired columns. Heretofore, it has been assumed that they had been used to decorate the windows of the chapel and capital hall in the northern wing of the main castle. Actually they originate from a structure which had an open gallery or cloister. However, this would mean that there was a very richly decorated structure in $13^{\text {th }}$ century Old Livonia that was demolished less than 50 years after it was built and the ruins were dispersed. There were very few such structures in Old Livonia at the time. However, one such case does exist, and it is not far from Viljandi - namely the Old Pärnu Cathedral.

The main church of the Oesel-Wiek bishopric in Old Pärnu was completed in the early 1250s. Based on written records, we know that there was a capital hall, refectory and dormitory for the cathedral chapter house. This spatial plan also alludes to the existence of a cloister. The Old Pärnu Cathedral was destroyed by the Lithuanians in 1263. Later, the ruins of the cathedral were reconstructed into a parish church. However, this means that the cloister was no longer need. And it is possible that some of the carved decorations were transported to Viljandi, where the construction of a large-scale castle was under way during the last decades of the $13^{\text {th }}$ century. 


\section{CV}

Kaur Alttoa (b. 1947) has been a long-time scholar and teacher of art history at the University of Tartu and he is one of the foremost specialists of the history of medieval architecture in the Baltic region. His objects of interest have included both medieval fortresses and sacral buildings. Among his major contributions stands out the restoration and study of the St John's Church in Tartu, which was also published as a monograph in 2011. 